\title{
Liver slice culture as a model for lipid metabolism in fish
}

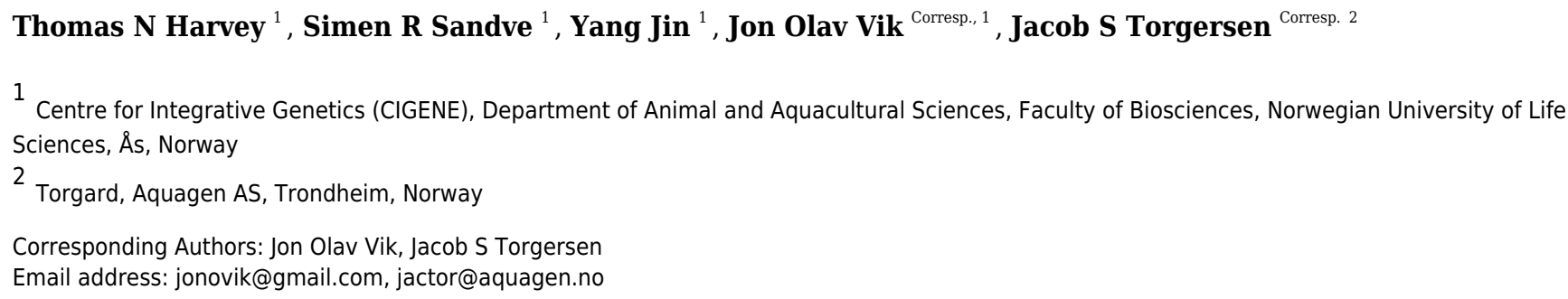

Hepatic lipid metabolism is traditionally investigated in vitro using hepatocyte monocultures lacking the complex three-dimensional structure and interacting cell types essential liver function. Precision cut liver slice (PCLS) culture represents an alternative in vitro system, which benefits from retention of tissue architecture. Here we present the first comprehensive evaluation of the PCLS method in fish (Atlantic salmon) and validate it in the context of lipid metabolism using feeding trials, extensive transcriptomic data, and fatty acid measurements. We observe an initial period of post-slicing global transcriptome adjustment, which plateaued after three days in major metabolic pathways and stabilized through nine days. PCLS fed alpha-linolenic acid (ALA) and insulin responded in a liver-like manner, increasing lipid biosynthesis gene expression. We identify interactions between insulin and ALA, where two PUFA biosynthesis genes that were induced by insulin or ALA alone, were highly down-regulated when insulin and ALA were combined. We also find that transcriptomic profiles of liver slices are exceedingly more similar to whole liver than hepatocyte monocultures, both for lipid metabolism and liver marker genes. PCLS culture opens new avenues for high throughput experimentation on the effect of "novel feed composition" and represent a promising new strategy for studying genotype-specific molecular features of metabolism. 


\section{Liver slice culture as a model for lipid metabolism in fish}

2 Thomas N. Harvey', Simen R. Sandve', Yang Jin', Jon Olav Vik'*, Jacob S. Torgersen*

3

4 'Centre for Integrative Genetics (CIGENE), Department of Animal and Aquacultural Sciences, Faculty of Biosciences,

5 Norwegian University of Life Sciences, NO-1432 Ås, Norway

$6 \quad{ }^{2}$ AquaGen AS, Postboks 1240, Torgard, NO-7462 Trondheim, Norway

$7 \quad *$ shared corresponding author

8

9 Corresponding authors:

10 Jon Olav Vik, Ph.D., Centre for Integrative Genetics (CIGENE), Department of Animal and Aquacultural

11 Sciences, Faculty of Biosciences, Norwegian University of Life Sciences, NO-1432 Ås, Norway,

12 jonovik@gmail.com,+47 45882998

13 Jacob S. Torgersen, Ph.D., AquaGen AS, Postboks 1240, Torgard, NO-7462 Trondheim, Norway, 14 jactor@aquagen.no,+4792019966 


\section{Abstract}

16 Hepatic lipid metabolism is traditionally investigated in vitro using hepatocyte monocultures lacking the

17 complex three-dimensional structure and interacting cell types essential for liver function. Precision cut 18 liver slice (PCLS) culture represents an alternative in vitro system, which benefits from retention of tissue 19 architecture. Here we present the first comprehensive evaluation of the PCLS method in fish (Atlantic salmon) and validate it in the context of lipid metabolism using feeding trials, extensive transcriptomic data, and fatty acid measurements. We observe an initial period of post-slicing global transcriptome adjustment, which plateaued after three days in major metabolic pathways and stabilized through nine days. PCLS fed alpha-linolenic acid (ALA) and insulin responded in a liver-like manner, increasing lipid biosynthesis gene expression. We identify interactions between insulin and ALA, where two PUFA biosynthesis genes that were induced by insulin or ALA alone, were highly down-regulated when insulin and ALA were combined. We also find that transcriptomic profiles of liver slices are statistically more similar to whole liver than hepatocyte monocultures after 5 days, both for lipid metabolism and liver marker genes. PCLS culture opens new avenues for high throughput experimentation on the effect of "novel feed composition" and represent a promising new strategy for studying genotype-specific molecular features of metabolism.

\section{Introduction}

Liver is the metabolic transformation hub. It is responsible for receiving nutrients absorbed in the gut through the portal vein, processing these nutrients for storage or energy production, and subsequent transportation of metabolic products throughout the body. Essential to proper function, the complex threedimensional structure of liver consists of intrahepatic microcirculatory units (lobules) of tightly associated cells that communicate through paracrine and autocrine effects (Wake \& Sato, 2015). The liver is also the main organ for detoxification, so in vitro methods are commonly applied for toxicological studies to reduce use of in vivo experiments. Hepatocyte cultures were established in the 1970s (Ekins, 1996), and rapidly became the preferred model system for toxicology. Liver slice culture was first introduced in 1923 
41 The development of automated tissue slicers in the 1980s (Krumdieck, Dos Santos \& Ho, 1980) solved this

42 problem, so liver slices became a viable option. One of the main advantages of liver slices is the retention

43 of normal cell composition and 3D structure. In addition, the preparation is fast, reproducible, without

44 enzymatic cell dissociation, and no need for coating the growth surface. Together with established

45 protocols, this has heralded the return of liver slices for in vitro studies.

Precision cut liver slice (PCLS) cultures have been applied in a number of toxicology studies and most recently also immunology (Wu et al., 2018), however the use of PCLS to study central liver metabolism is sparse, with few PCLS studies investigating aspects of lipid metabolism, all of which are in mammals (Neyrinck, Gomez \& Delzenne, 2004; Szalowska et al., 2014; Janssen et al., 2015; Fortin et al., 2017). We provide a critical evaluation of PCLS as a metabolic model system in fish by characterizing whole transcriptome changes in the context of lipid metabolism. We chose Atlantic salmon for its economic importance; and because development of feeding and breeding strategies that optimize omega-3 production require a better understanding lipid metabolism. Additionally, the effect of altered feed fatty acid profile on making this an ideal system for assessing the effects of altering media fatty acid composition and comparing to expected in vivo gene expression.

Here we integrate transcriptomics data with domain knowledge to describe a method for using PCLS as a model system to study lipid metabolism. We aim to 1) characterize transcriptome wide changes in liver

61 slice culture over time, 2) demonstrate the utility of using liver slice culture to study lipid metabolism, and

62 3) compare gene expression patterns between liver slice culture, $2 \mathrm{D}$ hepatocyte culture, and whole liver in vivo.

\section{Materials and Methods}


66

67

68

69

\section{Liver slice culture}

Atlantic salmon used in this study were sacrificed according to the Norwegian Animal Research Authority (NARA); regulations for use of experimental animals (FOR-2015-06-18-761). The liver was removed immediately after euthanization and placed in ice cold Hank's balanced salt solution (HBSS, Thermofisher). Livers were cut into approximately $4 \mathrm{~mm} \times 4 \mathrm{~mm} \times 8 \mathrm{~mm}$ strips before being superglued to a plastic piston and encased in ultra-low melt agarose (Merck). Liver strips were sliced to a thickness of $300 \mu \mathrm{m}$ using a compresstome VF-300 (Precisionary Instruments) and collected in ice cold HBSS before being transferred to $15^{\circ} \mathrm{C}$ Leibovitz 15 medium $\mathrm{pH} 7.4$ (L15, Thermofisher) containing 5\% fetal bovine serum (FBS, Merck) and $1 \%$ penicillin - streptomycin (PS, Thermofisher) which will now be referred to as base media. Liver slices were incubated in sterile $6(2 \mathrm{~mL}$ media per well $)$ or $12(4 \mathrm{~mL}$ media per well $)$ well cell culture plates with netwell inserts (Corning, $500 \mu \mathrm{m}$ membrane size) for up to 9 days at $15^{\circ} \mathrm{C}$ under ambient air. For each of the following experiments, liver slices were prepared from a single fish each time to eliminate biological variation.

\section{Time course experiments}

We performed two time course experiments, the first to test the effect of culturing time on the liver slices, and the second as a follow up to test the effect of media change frequency and inclusion of insulin over time. In both experiments, liver slices were generated immediately after euthanization and viability measurements were taken every day in the first experiment and on days three and six in the second experiment. All samples were stored in RNAlater at $-20^{\circ} \mathrm{C}$. In the first experiment we generated slices from a saltwater life-stage Atlantic salmon $(\sim 200 \mathrm{~g})$ reared on a marine oil based diet high in DHA and EPA. Immediately after euthanization, liver slices were generated as described above. Media was changed after sampling on days three and six using base media. On day three base media was supplemented with $700 \mu \mathrm{M}$ randomly methylated beta cyclodextrin (BCD) and $0.7 \%$ ethanol. Samples were taken in triplicate before slicing (whole liver) and 1, 3, 4, 5, 6, 7, 8, and 9 days after slicing. In the second time course experiment we used Atlantic salmon in the freshwater life-stage reared on a marine oil diet high in EPA and DHA. Human insulin (Merck) was included in the media at $20 \mathrm{nM}$ and media was refreshed either every day or 
92 every third day with fresh base media containing $20 \mathrm{nM}$ insulin. Samples were taken in triplicate before

93 slicing (whole liver) and days 3, 4, 5, 6, 7, 8, and 9.

\section{Fatty acid and insulin gradient experiments}

95 We performed two concentration gradient experiments, the first was used for transcriptomic analysis, the

96

second for fatty acid analysis. In the first experiment liver slices were prepared from two freshwater stage Atlantic salmon $(\sim 50 \mathrm{~g})$, one for use in the fatty acid gradient experiment and one for use in the insulin gradient experiment. We used randomly methylated beta-cyclodextrin (BCD) as our fatty acid delivery system since it has been demonstrated to efficiently deliver fatty acids across membranes in other in vitro systems (Brunaldi, Huang \& Hamilton, 2010). Alpha-linolenic acid (ALA) was stored at 10mM in ethanol then mixed 1:1 with $100 \mathrm{mM}$ BCD in water for a final molar ratio of 1:10 fatty acid to BCD. From this stock ALA was added to the media at a concentration of 0 ( $700 \mu \mathrm{M}$ BCD only), $20,40,70$, and $100 \mu \mathrm{M}$, aliquoted into a new six well culture plate, and placed at $15^{\circ} \mathrm{C}$ to equilibrate for at least 30 minutes. For all ALA treated samples, liver slices were transferred to ALA supplemented base media after a three day recovery period. For the insulin containing samples, human insulin (Merck) was diluted in base media to a final concentration of 10 or $100 \mathrm{nM}$ and incubated with liver slices from the beginning of the experiment. All liver slices were sampled in triplicate on day five and stored in RNAlater at $-20^{\circ} \mathrm{C}$. In the second concentration gradient experiment, liver slices were prepared from freshwater stage fish $(\sim 500 \mathrm{~g})$ and supplemented with $0(700 \mu \mathrm{M}$ BCD only), 20, 40, 70, 100, and $140 \mu \mathrm{M}$ ALA on day three as described except this time ethanol was evaporated under a stream of nitrogen before mixing with BCD. Samples were taken in triplicate on day four, washed in ice cold HBSS, flash frozen in an ethanol dry ice slurry, and stored at $-80^{\circ} \mathrm{C}$.

\section{D hepatocyte culture experiment}

Primary cells were isolated from salmon liver as described (Bell et al., 1997), with some modifications. After euthanization, the liver was removed and rinsed in ice cold $\mathrm{Mg} 2+/ \mathrm{Ca} 2+$ free $\mathrm{HBSS}$, before $\sim 100 \mathrm{ml}$ of the same buffer was directly injected with a $50 \mathrm{ml}$ syringe and $27 \mathrm{G}$ needle, at various places to wash out blood cells. Then, $30 \mathrm{ml}$ of HBSS with 150U/ml Collagenase type 1 (Merck) was injected, before the tissue

Peer] reviewing PDF | (2019:01:34550:2:0:NEW 13 Aug 2019) 
118 was finely chopped. The tissue suspension was incubated for $1 \mathrm{~h}$ at $10-12^{\circ} \mathrm{C}$ with agitation. Dissociated cells

119 were collected by cell straining $(70 \mu \mathrm{m})$ and centrifugation for $10 \mathrm{~min}$ at $100 \mathrm{~g}$. After three washes in HBSS,

120 the pellet was resuspended in base media supplemented with $10 \mu \mathrm{M}$ human insulin (Merck) and grown at

$121200 \mathrm{k} / \mathrm{cm}^{2}$ density at $15^{\circ} \mathrm{C}$ on polyethyleneimine coated wells (Vancha et al., 2004). Cells were

122 supplemented with ALA on day five as previously described and collected in triplicate using a cell scraper

123 on days five (before ALA), six, and eight by flash freezing and storing at $-80^{\circ} \mathrm{C}$.

\section{Viability measurement}

125 Slice viability was assessed by staining with Hoechst and propidium iodide to identify live and dead cells.

126 Slices were transferred to L15 medium containing $10 \mu \mathrm{g} / \mathrm{mL}$ Hoechst and $10 \mu \mathrm{g} / \mathrm{mL}$ propidium iodide for 5

127 minutes at $15^{\circ} \mathrm{C}$. Slices were then transferred to fresh $\mathrm{L} 15$ medium and placed on ice until being imaged

128 with a scanning laser confocal microscope (CLSM, Leica). Live/dead ratios were determined using Icy

129 (http://www.bioimageanalysis.org/). We compared the proportions of live and dead cells in two randomly

130 selected locations per slice to determine overall slice viability.

\section{Microscopy}

132 We made cross sections of liver slices at three different time points during culturing (one slice per time point) and observed morphological changes using light microscopy. All samples for microscopy were fixed using $4 \%$ formalin in phosphate buffered saline (PBS) for 1 hour then transferred to $70 \%$ ethanol stepwise (PBS-25\%-50\%-70\%) for 5 minutes at each step and stored at $-20^{\circ} \mathrm{C}$ until microscopic analysis was performed. Prior to paraffin embedding liver slices were transferred to $96 \%$ ethanol stepwise (70\%-85\%96\%-96\%) for 5 minutes at each step then washed twice with histoclear (National diagnostics) for 5 minutes each. Next, liver slices were embedded in paraffin (Merck) by incubating in paraffin at $61^{\circ} \mathrm{C}$ three times for 10 minutes each. Paraffin was allowed to solidify at room temperature. Liver slice cross-sections were 140 prepared using a rotary microtome (Leica) at a thickness of $7 \mu \mathrm{m}$, placed on the surface of a $43^{\circ} \mathrm{C}$ water

141 bath, and floated onto a clean microscopy slide. Sections were deparaffinized by washing twice with 142 histoclear for 5 minutes each and rehydrated by transferring to $70 \%$ ethanol stepwise (histoclear-96\%-85\%$14370 \%-70 \%$ ) for 5 minutes each followed by a brief wash in distilled water. Sections were stained with a $1 \%$ 
144 hematoxylin solution (Mayer's) for eight minutes, rinsed in running tap water for 10 minutes followed by

$14596 \%$ ethanol and counterstained with a $0.25 \%$ eosin-phloxine B solution for 30 seconds. Stained sections

146 were washed twice with histoclear for five minutes each and mounted with DPX (Merck). Micrographs of

147 cross-sections were taken at 20x magnification on a light microscope (Leica).

\section{RNA sequencing}

149 Slices were stored in RNAlater (Merck) at $-20^{\circ} \mathrm{C}$ until RNA extraction using the RNeasy universal kit

150 (QIAGEN). RNA concentration was determined on a Nanodrop 8000 and quality was determined on an

151 Agilent 2100 bioanalyzer using Agilent RNA 6000 nano chips. All RNA samples had a RNA integrity

152 number greater than 7. mRNA libraries were prepared using the Trueseq library preparation kit (Agilent).

153 Concentration and mean length were determined by running cDNA libraries on a bioanalyzer 2100 using

154 DNA 1000 chips (Agilent). RNA libraries were sequenced on an Illumina HiSeq 2500 with 100-bp single

155 end reads.

\section{RNAseq analysis}

157 All RNA sequencing and demultiplexing was done at the Norwegian sequencing center (Oslo, Norway).

158 Fastq files were trimmed and mapped the the salmon genome (ICSASG_v2) using STAR (v2.5.2a) (Dobin

159 et al., 2013). Mapped reads for each gene were counted with HTSeq-count (v0.6.1p1) (Anders, Pyl \&

160 Huber, 2015). Differential expression analysis was performed in R (v3.2.5) using the edgeR package

161 (Robinson, McCarthy \& Smyth, 2010). All counts were normalized to library size using TMM

162 normalization within edgeR. For the time course and gradient experiments an ANOVA-like differential

163 expression test was used to find difference between any of the conditions (see edgeR manual). This yielded

$164 \log 2$ fold change to the reference level (day0 or ALA0) and false discovery rate (FDR) for each gene. For

165 the time course experiments we considered genes with a FDR of $<0.01$ and $\log 2 \mathrm{FC}>1$ as differentially

166 expressed while for the gradient experiments genes with a FDR of $<0.01$ were considered differentially

167 expressed. Gene expression clusters were generated by applying wardD2 hierarchical clustering to gene-

168 scaled mean counts per million. KEGG enrichment was performed on each gene cluster using edgeR.

169 Pathways with a p-value $<0.001$ were considered significantly enriched. To compare gene expression 
170 between whole liver, liver slice, and hepatocyte culture, we pooled data from each source to give an overall

171 expression phenotype. Data on whole liver was obtained from a previously published feeding trial (Gillard

172 et al., 2018) and whole liver samples taken before generating liver slices. Data on liver slice and hepatocyte

173 culture was obtained from the previously described experiments.

\section{Lipid analysis}

175 Fatty acid methyl esters (FAME) were prepared from liver slices according to established protocols

176 (O'Fallon et al., 2007) with half volumes to account for the small size of liver slices. We used 13:0, 19:0,

177 and 23:0 as an internal standards in all samples and FAMEs were separated by gas chromatography on a

178 Trace GC Ultra (Thermo Scientific) using a flame ionization detector. Relative fatty acid abundance was

179 calculated from the resulting chromatograms.

\section{Statistical analysis}

181 All statistical analysis was performed in R (v3.2.5). Correlation analysis between whole liver and liver slice samples was calculated using the mean counts per million (CPM) of each gene across the three time course experiments (whole liver) and triplicate samples within each experiment (liver slice) for each day followed and insulin gradient experiments was calculated using a one-way analysis of variance (ANOVA) test followed by a Tukey-HSD test. Differences with a p-value $<0.05$ were considered significant.

\section{Results}

\section{Viability and morphology}

190 Liver slices had a mean viability of $90.3 \pm 2.7 \%$ (Fig. 1B). We did not observe any viability effects of ALA, 191 insulin nor methyl- $\beta$-cyclodextrin (BCD) used as a lipid carrier in the experiments. More generally, we find that viability at the end of an experiment is similar to the viability at the beginning of an experiment (Fig. 1B). This implies that preparation of the slices is most critical to viability, as opposed to culture time. Morphological analysis of liver slice cross-sections did not reveal any large change in the thickness of slices 
196

197

dehydration causing the liver slices to shrink during the paraffin embedding process (Fig. 1C). Slices examined were approximately $188 \mu \mathrm{m}, 183 \mu \mathrm{m}$, and $210 \mu \mathrm{m}$ in thickness on days 0,3 , and 5 , respectively.

\section{Time course experiments}

In order to study how the liver slices change in culture over time, we sequenced RNA from three experiments lasting for nine days. In time course one, media was changed every three days and samples were taken before slicing (day 0) and 1, 3, 4, 5, 6, 7, 8, and 9 days after slicing. On day three, slices were fed a control diet consisting of BCD only. We use BCD to deliver the fatty acids to the cells, so in this case $\mathrm{BCD}$ only was used as a control for fatty acid supplementation conditions. The second and third time course experiments differed from the first in terms of media change frequency (daily or every third day) and inclusion of insulin in the media $(20 \mathrm{nM})$.

To characterize the behavior of liver slices over time under control conditions, we performed ANOVA-like differential expression analysis testing for differentially expressed genes between any of the time points in time course one. This yielded 16,267 differentially expressed genes (DEG) with a false discovery rate $(\mathrm{FDR})<0.01$ and $\log 2$ fold change $(\log 2 \mathrm{FC})>1$ (Fig. 2A). We used hierarchical clustering to group genes with a similar expression trend into eight gene clusters (Fig. 2B), then searched for enriched pathways from the Kyoto encyclopedia of genes and genomes $(\mathrm{KEGG})$ in each of these clusters $(\mathrm{p}<0.0001)$ to characterize the overall trend of various physiological and metabolic processes (Fig. 2C). Interestingly, almost all pathways related to protein, lipid, carbohydrate and vitamin metabolism belong to clusters two and three, which decreased between day zero (before slicing) and day three, followed by an overall stabilization in expression through day nine. Pathways related to signal transduction were mostly enriched in clusters seven and eight, which increased expression greatly between day zero and day one (before and 24 hours after slicing), then decreased to original levels by day 9. Pathways related to cell growth and death were mostly enriched in clusters four, five, and six, which in general increased during 9 days of liver slice culture. 
221 Since cell culture aims to mimic the conditions and behavior of tissue in vivo, we compared gene expression

222 patterns between whole liver and liver slices for all three experiments. To assess the similarity in expression

223 patterns over time we calculated Spearman co-expression correlations between mean whole liver gene

224 expression and gene expression from each day in three time course experiments for all genes and genes

225 within seven relevant lipid metabolism pathways (Fig. 3). For time course one, correlation between liver

226 slices and whole liver decreased gradually over time from 0.90 on day 1 to 0.83 on day 4 , then stabilized

227 around 0.8 through day 9. A similar effect was observed in time course two and time course three with co-

228 expression correlation to whole liver stabilizing around 0.82 through day 8 then decreasing to 0.78 and 0.79

229 respectively on day 9 (Fig. 3). The greatest difference between whole liver and liver slices was in the

230 pathway "Steroid biosynthesis" with co-expression correlations hovering between 0.48 and 0.28 during

231 days three through nine for all three experiments, the result of upregulation of nearly all steroid biosynthesis

232 genes in liver slices. Co-expression correlation was slightly more stable over time when media was

233 refreshed daily, especially "Steroid biosynthesis"; however, overall expression similarity to whole liver was

234 high for nearly all pathways and time points examined.

\section{Fatty acid and insulin gradient experiments}

236 In order to evaluate fatty acid uptake and transcriptomic response in liver slices, we added alpha-linolenic acid (ALA) to the media in increasing concentrations from $20 \mu \mathrm{M}$ up to $100 \mu \mathrm{M}$. We expect this to trigger upregulation of lipid metabolism-related gene expression as observed in liver of fish fed vegetable oil diets high in ALA (Gillard et al., 2018). ANOVA-like differential expression analysis testing for differences between any of the ALA concentrations yielded 8,282 DEGs (FDR $<0.01$, Fig. S1A). We then broke these DEGs into four expression clusters as previously explained (Fig. S1B). KEGG enrichment analysis on these four clusters yielded 37 total pathways significantly enriched $(\mathrm{p}<0.001)$ in one or more cluster (Fig. S1C).

We found that all enriched pathways relating to lipid metabolism belonged to the same cluster which increased with increasing ALA concentration, especially between 40 and $70 \mu \mathrm{M}$. Specifically, the pathways biosynthesis", and "PPAR signaling pathway" were all enriched in this cluster (Fig. S1B and C). 
248 In order to better characterize the effect of ALA supplementation on PUFA biosynthesis, we analyzed

249 individual gene expression of key genes in the PUFA biosynthesis pathway (Fig. 4). The five key genes

250 involved in PUFA biosynthesis that are differentially expressed at some point in the ALA concentration

251 gradient include delta-5 desaturase ( $\triangle 5 f a d)$, delta-6 desaturase a ( $\triangle 6 f a d a)$, fatty acid elongase 2 (elovl2),

252 fatty acid elongase $5 \mathrm{a}($ elovl $5 a)$, and fatty acid elongase $5 \mathrm{~b}($ elovl $5 b)$. All five genes displayed an overall

positive correlation with ALA concentration (Fig. 4B) with $\Delta 5 \mathrm{fad}, \Delta 6 \mathrm{fada}$, and elovl2 responding strongly

to ALA between $40 \mu \mathrm{M}$ and $70 \mu \mathrm{M}$ and both elovl5 genes less influenced, slightly increasing with increasing

ALA concentration. Counterintuitively, at low ALA concentration $(20 \mu \mathrm{M})$ all genes except elovl2 and elovl5a significantly decreased $(\mathrm{q}<0.05)$ in expression compared to control samples (no fatty acid). Between $70 \mu \mathrm{M}$ and $100 \mu \mathrm{M}$ ALA, expression of $\triangle 5 f a d, \Delta 6 f a d a$, and elovl2 did not significantly change (Fig. 4B).

To assess the impact of ALA supplementation (up to $140 \mu \mathrm{M}$ ) on the fatty acid profile of liver slices we conducted a second ALA concentration gradient experiment. As expected, percent ALA increased with increasing media ALA concentration from $0.87 \%$ with no ALA supplementation to $6.5 \%$ with $140 \mu \mathrm{M}$ ALA supplementation (Fig. 4C). In addition, the elongation product of ALA, 20:3n-3, increased with increasing media ALA concentration from $0.35 \%$ with no ALA supplementation to $2.7 \%$ with $140 \mu \mathrm{M}$ ALA supplementation (Fig. 4C). EPA and DHA levels do not significantly $(q<0.05)$ change at any point in the $29.6 \%$ to $25.1 \%$, respectively (table $\mathrm{S} 1$ ).

The effect of insulin supplementation on liver slices was assessed by incubating slices with two different concentrations of insulin, $10 \mathrm{nM}$ and $100 \mathrm{nM}$. To test for an interaction between insulin and fatty acid 
272 supplementation, we also tested these insulin levels with and without supplementation of $70 \mu \mathrm{M}$ ALA.

273 Differential expression analysis testing for changes in expression between any of the conditions (without

274 ALA supplementation) yielded 11,898 DEGs (FDR $<0.01$, Fig. S2A). Approximately half of these genes

275 were upregulated (5,889 DEGs) and half were downregulated (6,012 DEGs) regardless of insulin 276 concentration (Fig. S2B). Only 13 genes were differentially expressed between the two insulin

277 concentrations. KEGG pathway enrichment on these gene clusters revealed that most metabolism related 278 pathways were upregulated with the addition of insulin. Specifically relating to lipid metabolism, 279 "biosynthesis of unsaturated fatty acids", "fatty acid biosynthesis", and "PPAR signaling pathway" were 280 significantly enriched in the upregulated gene set (Fig. S2C). Pathways related to metabolism enriched in 281 the downregulated gene set included "glycerophospholipid metabolism", "inositol phosphate metabolism", 282 and interestingly "insulin signaling pathway” (Fig. S2C).

Insulin supplementation alone tended to increase expression of key PUFA biosynthesis genes except for $\triangle 5 f a d$, which did not significantly $(\mathrm{q}<0.05)$ change with increasing insulin concentration (Fig. 4D). Increasing insulin concentration from $10 \mathrm{nM}$ to $100 \mathrm{nM}$ did not significantly change the expression of any of the five genes. Addition of $70 \mu \mathrm{M}$ ALA had a large effect on the expression of $\Delta 5 \mathrm{fad}$ and $\Delta 6 \mathrm{fada}$, which were expressed most in the absence of insulin, then downregulated upon insulin supplementation. ALA supplementation did not appear to have a large effect on the expression of elovl $5 a$ and elovl $5 b$, which agrees with findings from the ALA gradient experiment.

\section{Liver slice culture versus primary cell culture}

292 To assess how liver slice culture compares to widely used hepatocyte culture and liver in vivo, we compared RNA sequencing data from hepatocyte culture $(n=16$ from one fish), liver slice culture $(n=89$ from four fish), and whole liver ( $\mathrm{n}=210$ from 210 fish). Hepatocytes were sampled after 5, 6, and 8 days in culture, so only liver slices incubated at least 5 days were used for comparison. The hepatocytes displayed a cuboidal 
298 hepatocytes remained healthy after 8 days in culture. Data on whole liver was obtained from a feeding trial 299 where salmon were fed either a fish oil or plant oil based diet (Gillard et al., 2018). All data from each 300 source was pooled to give a range of possible expression patterns from that source. We then calculated 301 relative expression by scaling expression of each gene across all data sources.

302

303 We find that overall, liver slice culture more closely resembles whole liver than hepatocyte culture. Gene 304 expression in the lipid related KEGG pathways "fatty acid biosynthesis", "glycerolipid metabolism",

"biosynthesis of unsaturated fatty acids", and "steroid biosynthesis" was much lower in hepatocyte culture

relative to both liver slice culture and whole liver (Fig. 5A). This was reflected in the expression of all underlying key PUFA biosynthesis genes except elovl5a (Fig. 5B). Expression of genetic marker genes characteristic of functional liver was generally lower in hepatocyte culture, while liver slice culture was closer, but not identical to whole liver expression patterns (Fig. 5C). Specifically, albumin genes alb1, alb21, and alb2-2 and glucose-6-phosphatase genes g6pcl-3 and g6pcl-4 had lowest expression in hepatocyte culture followed by liver slice culture and highest expression in whole liver (Fig. 5C).

\section{Discussion}

\section{Liver slice metabolism stabilizes after three days and remains liver-like} 315 through nine days in culture

316 We find that time, up to 9 days tested, does not have a large effect on the viability or thickness of the slices.

317 This is encouraging, since morphometric analysis of Atlantic cod liver slices showed an increase in the 318 proportion of dead cells at 72 hours in culture (Eide et al., 2014) and studies on rat liver slices have shown 319 that changes in viability and slice thickness over time is highly dependent on the culture media used 320 (Starokozhko et al., 2015).

322 We do, however, observe a time dependent drift in gene expression patterns. Slices most resemble whole 323 liver 24 hours after slicing with a correlation coefficient of 0.90 and gradually decrease in similarity over 
324 time (Fig. 3). A similar effect has been observed in rat liver slices (Boess et al., 2003), however, the rate

325 that slices diverged from whole liver was much lower in our experiments. High correlation ( $>0.8)$ to whole

326 liver was maintained through day four in time course one and through day six in time courses two and three.

327 The observed decrease in co-expression correlation in the pathway "steroid biosynthesis" was due to

328 upregulation of nearly all steroid biosynthesis genes three days after slicing and is probably due to a

329 deficiency of cholesterol in the media. BCD is known to dose dependently remove cholesterol from cell

330 membranes in culture, however since samples were taken before BCD was added on day three and co-

331 expression correlation decreases on day 3 (before BCD exposure), it is unlikely that BCD is causing this

332 effect. Genes that are highly upregulated 24 hours after slicing were mostly enriched in signaling pathways

333 (Fig. 2), likely related to repair and inflammatory response processes known to be triggered by physical

334 liver damage that is unavoidable during the slicing process ( $\mathrm{Su}$ et al., 2002). Since metabolic gene 335 expression stabilizes after three days (Fig. 2, clusters 2 and 3), we used a three day recovery period for

336 future metabolic studies so that changes in gene expression are more likely to be the result of the treatment

337 rather than time. The gradual downward trend in co-expression correlation over time represents a slow drift

338 in the global gene expression phenotype as opposed to a rapid gene expression change upon hepatocyte

339 culturing. This is a known problem with hepatocyte cultures resulting from a combination of factors,

340 especially the lack of circulating hormones produced elsewhere in the body causing time-dependent de-

341 differentiation of hepatocytes (Elaut et al., 2006). Expression of liver marker genes was markedly higher in

342 our liver slice culture than 2D hepatocyte culture (Fig. 5C) representing an improvement in long-term

343 hepatocyte stability.

\section{Exogenous ALA is taken up and triggers a liver-like response}

345 ALA complexed with BCD was efficiently delivered to cells in a dose dependent manner. We observed a

346 proportional increase in ALA and 20:3n-3 with increasing ALA concentration. While the ALA increase

347 could be due to residual ALA sticking to the cells from the media, the proportional increase in 20:3n-3 with

348 media ALA concentration supports active uptake and elongation of exogenous fatty acids (Fig. 4C). There

349 was no significant change in EPA or DHA after ALA supplementation. This does not mean that EPA and 
350 DHA are not being produced, but rather that the amount of ALA in the media is too low to cause a 351 measurable increase in the already abundant pool of EPA and DHA in the cells. Indeed, both ALA and $352 \quad 20: 3 n-3$ are low ( $0.88 \%$ and $0.35 \%$ respectively) in control slices, so a small increase in abundance could 353 be detected. Future studies feeding radiolabeled ALA to liver slices are needed to confirm the production 354 of DHA and EPA in PCLS.

ALA fed to slices has two fates within the PUFA biosynthesis pathway. The first and most common is the canonical pathway, where ALA is first desaturated by a $\Delta 6$ desaturase to $18: 4 n-3$, then elongated and desaturated to EPA and DHA via Sprecher's shunt (Voss et al., 1991). The second occurs when ALA is first elongated to $20: 3 n-3$, presumably by ELOVL5. In this case, a $\Delta 8$ desaturase is required to form $20: 4 n-$ 3, which can then continue to EPA and DHA via the canonical pathway. This does however not happen efficiently in Atlantic salmon because of the low $\Delta 8$ desaturase activity of $\Delta 6 \mathrm{FADb}$ (Monroig, Li \& Tocher, $2011)$ in combination with low expression in liver (0.5-3.2 counts per million). Rather, 20:3n-3 accumulates in the cells or is catabolized for energy (Tocher, 2003), which is consistent with observations in feeding trials where fish fed vegetable oil based diets high in ALA contained higher tissue levels of 20:3n-3 (Tocher et al., 2001; Bell et al., 2010). This can explain why we measure increased levels of 20:3n-3, but not other PUFA intermediates.

We also observe that 18:0 increases between whole liver and liver slices. This corresponds with increased expression of both both fatty acid synthase genes, fasa and fasb, which doubled (log2FC 1.08 and 1.02, respectively) in expression one day after slicing and the pathways "fatty acid biosynthesis" and "glycerolipid metabolism" were enriched in clusters that spike one day after slicing (clusters two and seven, Fig. 2C). Conversely, high levels of saturated fatty acids are known to be toxic to cells, so to avoid this cells rapidly desaturate 18:0 to $18: 1 \mathrm{n}-9$, but we observe a decrease in this fatty acid in slices. This suggests that de novo lipogenesis is not responsible for the increase in 18:0. Upregulation of fas could be stress 
376 and injury (Groves et al., 2017). It is also unlikely that the 18:0 originates from FBS added to the media,

377 since previous studies have found that FBS also contains high levels of 18:1n9 and we find that this

378 decreases in liver slices (Stoll \& Spector, 1984). In addition, we found that as media ALA concentration

379 increased, total fat in liver slices decreased (table S2). This corresponds with BCD concentrations of $700 \mu \mathrm{M}$

380 in control slices $(0 \mu \mathrm{M}$ ALA) and between $200 \mu \mathrm{M}(20 \mu \mathrm{M}$ ALA) up to $1400 \mu \mathrm{M}$ (140M ALA). Since BCD

381 concentrations are the same in control slices and slices fed $70 \mu \mathrm{M}$ ALA, but total fat is lower in $70 \mu \mathrm{M}$ ALA

382 slices, it appears that this effect is related to the concentration of ALA rather than the concentration of BCD.

383 It is possible that feeding high levels of ALA to liver slices increases oxidative stress and induces lipolysis,

384 thus decreasing total fat in a dose dependent manner. Alternatively, ALA could stimulate lipoprotein

385 production in liver slices, similarly reducing total fat in slices. Future experiments could measure

386 lipoprotein formation and markers for oxidative stress in liver slices fed high levels of ALA to explain this 387 effect.

Overall, ALA concentration was positively correlated to lipid metabolism related gene expression, especially in PUFA biosynthesis with expression of all key pathway genes increasing with ALA. The same effect is known to occur in Atlantic salmon livers where fish fed vegetable oil-based diets high in ALA have higher PUFA biosynthesis gene expression relative to salmon fed fish oil-based diets low in ALA and high in EPA/DHA (Gillard et al., 2018). Additionally, this has been observed in vitro using Atlantic salmon primary hepatocytes (Kjær et al., 2016) and in vivo on rat liver (Tu et al., 2010). At very low concentration $(20 \mu \mathrm{M})$, expression of $\Delta 5 f a d, \Delta 6 f a d a$, and elovl $5 b$ actually decreased relative to control slices with no ALA supplementation. In this experiment samples were taken two days after exposure to ALA, so it is possible that in 48 hours all of the ALA in the media was depleted, presumably taken up by the cells and anabolized to longer chain fatty acid products that have an inhibitory effect on expression. Additionally, the "PPAR signaling pathway", which includes PPARs and target genes, was significantly enriched in cluster three which increases with increasing ALA concentration (Fig. S1). PPARs are well known transcriptional factors 
402 lipid metabolism (Poulsen, Siersbæk \& Mandrup, 2012). Taken together our results demonstrate the ability

403 of our PCLS model to accurately mimic expected shifts in lipid metabolism genes, highlighting its quality

404 as an in vitro system.

\section{Insulin triggers an anabolic response}

406 Lipid metabolism, like other metabolic processes, is highly influenced by the feed status of the fish with

407 insulin production triggered by feeding (Navarro et al., 2002). In order to ensure that the liver slices behaved

408 similarly to liver in fed fish, we assessed the inclusion of insulin in the media. A main function of insulin

409 is to shift the metabolic state from catabolic to anabolic, since it would be counterproductive for cells to

410 actively produce energy by breaking down organic macromolecules while at the same time storing energy

411 by building them up (Dimitriadis et al., 2011). In line with this we observe a binary response with several

412 thousand genes either upregulated or downregulated in the presence of insulin, regardless of concentration

413 (Fig. S2). Major anabolic pathways including "biosynthesis of unsaturated fatty acids" and "fatty acid

414 biosynthesis" are upregulated in the presence of insulin in agreement with an anabolic response.

415 Physiological range for circulating insulin is $0.2-5 \mathrm{nM}$ (Caruso \& Sheridan, 2011), so it is plausible that

416 raising insulin concentrations to $100 \mathrm{nM}$ has little effect because all of the insulin receptors are likely bound

417 at $10 \mathrm{nM}$.

\section{Insulin and ALA interact to regulate PUFA biosynthesis gene expression}

419 Insulin and ALA displayed complex interaction effects on expression of genes related to PUFA biosynthesis

420 in liver slices. Unaffected by ALA concentration, elovl5a and elovl5b were highly upregulated in the

421 presence of insulin. On the other hand, genes that were upregulated in response to ALA tended to be

422 upregulated in the presence of insulin alone, but then downregulated in the presence of insulin when

423 combined with ALA (Fig. 4D). An important regulator of lipid metabolism in liver, sterol regulatory

424 element binding protein 1 (srebp-1), is known to be upregulated by insulin through the PI3K/Akt/mTOR

425 signaling pathway (Matsuzaka \& Shimano, 2013), and indeed srebp-1 is upregulated in response to insulin

426 in our experiments. Both elovl5a and elovl $5 b$ contain sterol regulatory elements in their promoter regions

427 (Carmona-Antoñanzas et al., 2013), and along with $\Delta 6$ fada have been shown to increase in expression when 
co-transfected with srebp-1 (Carmona-Antonanzas et al., 2014). On the other hand, activation of PPARa

by ALA could work in opposition to insulin-mediated effects by stimulating beta-oxidation and

430 ketogenesis. There is evidence in rats that $\Delta 5$ desaturase (D5D) and $\Delta 6$ desaturase (D6D) are under dual

431 regulation by both SREBP-1 and PPARa (Matsuzaka et al., 2002), and given that regulation of lipid

432 metabolism is highly conserved across species (Carmona-Antonanzas et al., 2014) it is likely a similar effect

433 is present in salmon. The contrasting effect of insulin and ALA supplementation highlights the complex

434 interplay between signaling networks balancing hormonal and nutritional input to optimize regulation of

435 PUFA metabolism in Atlantic salmon.

\section{Liver slices are more suitable for long-term culture than primary hepatocytes}

437 We find that liver slices maintained liver-like gene expression patterns for longer than primary heptatocytes,

438 which are generally known to undergo time-dependent de-differentiation (Elaut et al., 2006). We attribute

439 this to the maintenance of the complex three-dimensional organization of whole liver with all interacting

440 cell types. While hepatocytes are generally responsible for the metabolic activities associated with liver,

441 regulation of these functions is controlled in concert with nonparenchymal cells through complex endocrine

442 and autocrine signaling networks (Kmiec, 2001). Eicosanoid signaling is a key component of these

443 networks, which represents a layer of information that is completely lost in 2D hepatocyte cultures since

444 eicosanoids are only produced in nonparenchymal cells (Johnston \& Kroening, 1996). In mammals, glucose

445 metabolism has been demonstrated to be influenced by nonparenchymal produced eicosanoids

446 (Cherrington, 1999) and there is evidence that regulation of lipogenesis and PUFA metabolism is influenced

447 by eicosanoid-mediated effects (Jump et al., 1999). In addition to eicosanoid production, interactions

448 between hepatocytes and nonparenchymal cells are known to play a role in cell proliferation and

449 differentiation (Kmiec, 2001) which could explain the higher liver slice culture expression of liver marker

450 genes. This, along with many other factors likely contribute to the observed long-term differences between

451 liver slice culture and 2D hepatocyte culture. Many of the metabolic processes in the liver are also regulated

452 by circulating hormones produced in other parts of the body, so while liver slice culture is not identical to 
453 whole liver, we find that liver slice culture is better suited to long term metabolic studies than primary 454 hepatocyte culture.

455

\section{Conclusion}

457 Taken together, our results demonstrate the utility and effectiveness of precision cut liver slices as a tool 458 for studying lipid metabolism in Atlantic salmon. We found that when studying metabolism in liver slices, it is best to allow the slices to recover for three days before adding fatty acids, since gene expression in pathways relating to metabolism remains stable after three days in culture. Liver slices were highly responsive to both exogenous fatty acids and insulin in line with current understanding of lipid metabolism of Atlantic salmon. Supplementation with ALA induced expression of lipid metabolism genes and pathways while supplementation with insulin shifted gene expression to an anabolic state as expected. We also observed a different, sometimes opposing, regulatory effect of insulin and ALA on expression of genes involved in PUFA biosynthesis. Liver slices mimic the complex three dimensional structure of the liver and produce results that are more relatable to liver in vivo than 2D hepatocyte culture. For this reason, liver slices are an attractive alternative to $2 \mathrm{D}$ hepatocyte culture for interrogating metabolic pathways.

\section{References}

Anders S, Pyl PT, Huber W. 2015. HTSeq--a Python framework to work with high-throughput sequencing data. Bioinformatics 31:166-169. DOI: 10.1093/bioinformatics/btu638.

Bell JG, Pratoomyot J, Strachan F, Henderson RJ, Fontanillas R, Hebard A, Guy DR, Hunter D, Tocher DR. 2010. Growth, flesh adiposity and fatty acid composition of Atlantic salmon (Salmo salar) families with contrasting flesh adiposity: Effects of replacement of dietary fish oil with vegetable oils. Aquaculture 306:225-232. DOI: 10.1016/j.aquaculture.2010.05.021.

Bell JG, Tocher DR, Farndale BM, Cox DI, McKinney RW, Sargent JR. 1997. The effect of dietary lipid on polyunsaturated fatty acid metabolism in Atlantic salmon (Salmo salar) undergoing parr-smolt 
transformation. Lipids 32:515-525. DOI: 10.1007/s11745-997-0066-4.

479

480

481

482

483

484

485

486

487

488

489

490

491

492

493

494

495

496

497

498

499

500

501

502

503

Boess F, Kamber M, Romer S, Gasser R, Muller D, Albertini S, Suter L. 2003. Gene Expression in Two Hepatic Cell Lines, Cultured Primary Hepatocytes, and Liver Slices Compared to the in Vivo Liver Gene Expression in Rats: Possible Implications for Toxicogenomics Use of in Vitro Systems. Toxicological Sciences 73:386-402. DOI: 10.1093/toxsci/kfg064.

Brunaldi K, Huang N, Hamilton JA. 2010. Fatty acids are rapidly delivered to and extracted from membranes by methyl-beta-cyclodextrin. Journal of lipid research 51:120-31. DOI: 10.1194/M900200-JLR200.

Carmona-Antonanzas G, Tocher DR, Martinez-Rubio L, Leaver MJ. 2014. Conservation of lipid metabolic gene transcriptional regulatory networks in fish and mammals. Gene 534:1-9. DOI: 10.1016/j.gene.2013.10.040.

Carmona-Antoñanzas G, Tocher DR, Taggart JB, Leaver MJ. 2013. An evolutionary perspective on Elovl5 fatty acid elongase: comparison of Northern pike and duplicated paralogs from Atlantic salmon. BMC Evolutionary Biology 13:85. DOI: 10.1186/1471-2148-13-85.

Caruso MA, Sheridan MA. 2011. New insights into the signaling system and function of insulin in fish. General and Comparative Endocrinology 173:227-247. DOI: 10.1016/j.ygcen.2011.06.014.

Cherrington AD. 1999. Control of glucose uptake and release by the liver in vivo. Diabetes 48:1198-214. DOI: 10.2337/DIABETES.48.5.1198.

Dimitriadis G, Mitrou P, Lambadiari V, Maratou E, Raptis SA. 2011. Insulin effects in muscle and adipose tissue. Diabetes Research and Clinical Practice 93:S52-S59. DOI: 10.1016/S01688227(11)70014-6.

Dobin A, Davis CA, Schlesinger F, Drenkow J, Zaleski C, Jha S, Batut P, Chaisson M, Gingeras TR. 2013. STAR: Ultrafast universal RNA-seq aligner. Bioinformatics 29:15-21. DOI: 10.1093/bioinformatics/bts635.

Eide M, Karlsen OA, Kryvi H, Olsvik PA, Goksøyr A. 2014. Precision-cut liver slices of Atlantic cod (Gadus morhua): An in vitro system for studying the effects of environmental contaminants. Aquatic 
Toxicology 153:110-115. DOI: 10.1016/j.aquatox.2013.10.027.

505 Ekins S. 1996. Past, Present, and Future Applications of Precision-Cut Liver Slices for in Vitro

506 Xenobiotic Metabolism. Drug Metabolism Reviews 28:591-623. DOI:

507 10.3109/03602539608994019.

Elaut G, Henkens T, Papeleu P, Snykers S, Vinken M, Vanhaecke T, Rogiers V. 2006. Molecular Mechanisms Underlying the Dedifferentiation Process of Isolated Hepatocytes and Their Cultures. Current Drug Metabolism 7:629-660. DOI: 10.2174/138920006778017759.

Fortin É, Blouin R, Lapointe J, Petit H V., Palin M-F. 2017. Linoleic acid, $\alpha$-linolenic acid and enterolactone affect lipid oxidation and expression of lipid metabolism and antioxidant-related genes in hepatic tissue of dairy cows. British Journal of Nutrition 117:1199-1211. DOI: 10.1017/S0007114517000976.

Gillard G, Harvey TN, Gjuvsland A, Jin Y, Thomassen M, Lien S, Leaver M, Torgersen JS, Hvidsten TR,

Groves JA, Maduka AO, O'Meally RN, Cole RN, Zachara NE. 2017. Fatty acid synthase inhibits the $O$ GlcNAcase during oxidative stress. Journal of Biological Chemistry 292:6493-6511. DOI: Vik JO, Sandve SR. 2018. Life-stage-associated remodelling of lipid metabolism regulation in 10.1074/jbc.M116.760785.

Janssen AWF, Betzel B, Stoopen G, Berends FJ, Janssen IM, Peijnenburg AA, Kersten S. 2015. The impact of PPAR $\alpha$ activation on whole genome gene expression in human precision cut liver slices. BMC Genomics 16:760. DOI: 10.1186/s12864-015-1969-3.

Johnston DE, Kroening C. 1996. Stimulation of prostaglandin synthesis in cultured liver cells by CCl4. Hepatology 24:677-684. DOI: 10.1002/hep.510240334.

Jump D., Thelen A, Ren B, Mater M. 1999. Multiple mechanisms for polyunsaturated fatty acid regulation of hepatic gene transcription. Prostaglandins, Leukotrienes and Essential Fatty Acids 60:345-349. DOI: 10.1016/S0952-3278(99)80010-6.

Kjær MA, Ruyter B, Berge GM, Sun Y, Østbye T-KK. 2016. Regulation of the Omega-3 Fatty Acid 

10.1371/journal.pone.0168230.

532

Kmiec Z. 2001. Cooperation of Liver Cells in Health and Disease. DOI: 10.1007/978-3-642-56553-3_7.

Krumdieck CL, Dos Santos JE, Ho K-J. 1980. A new instrument for the rapid preparation of tissue slices. Analytical Biochemistry 104:118-123. DOI: 10.1016/0003-2697(80)90284-5.

Leaver MJ, Bautista JM, Björnsson BT, Jönsson E, Krey G, Tocher DR, Torstensen BE. 2008. Towards Fish Lipid Nutrigenomics : Current State and Prospects for Fin-Fish Aquaculture. Reviews in Fisheries Science 16:73-94. DOI: 10.1080/10641260802325278.

Matsuzaka T, Shimano H. 2013. Insulin-dependent and -independent regulation of sterol regulatory element-binding protein-1c. Journal of diabetes investigation 4:411-2. DOI: 10.1111/jdi.12098.

Matsuzaka T, Shimano H, Yahagi N, Amemiya-Kudo M, Yoshikawa T, Hasty AH, Tamura Y, Osuga J, Okazaki H, Iizuka Y, Takahashi A, Sone H, Gotoda T, Ishibashi S, Yamada N. 2002. Dual regulation of mouse Delta(5)- and Delta(6)-desaturase gene expression by SREBP-1 and PPARalpha. Journal of lipid research 43:107-14.

Monroig Ó, Li Y, Tocher DR. 2011. Delta-8 desaturation activity varies among fatty acyl desaturases of teleost fish: High activity in delta-6 desaturases of marine species. Comparative Biochemistry and Physiology Part B: Biochemistry and Molecular Biology 159:206-213. DOI: 10.1016/J.CBPB.2011.04.007.

Navarro I, Rojas P, Capilla E, Albalat A, Castillo J, Montserrat N, Codina M, Gutiérrez J. 2002. Insights into Insulin and Glucagon Responses in Fish. Fish Physiology and Biochemistry 27:205-216. DOI: 10.1023/B:FISH.0000032726.78074.04.

Neyrinck AM, Gomez C, Delzenne NM. 2004. Precision-cut liver slices in culture as a tool to assess the physiological involvement of Kupffer cells in hepatic metabolism. Comparative hepatology 3 Suppl 1:S45. DOI: 10.1186/1476-5926-2-S1-S45.

O'Fallon J V., Busboom JR, Nelson ML, Gaskins CT. 2007. A direct method for fatty acid methyl ester synthesis: Application to wet meat tissues, oils, and feedstuffs. Journal of Animal Science 85:1511- 
Poulsen L la C, Siersbæk M, Mandrup S. 2012. PPARs: Fatty acid sensors controlling metabolism. Seminars in Cell \& Developmental Biology 23:631-639. DOI: 10.1016/J.SEMCDB.2012.01.003.

Robinson MD, McCarthy DJ, Smyth GK. 2010. edgeR: a Bioconductor package for differential expression analysis of digital gene expression data. Bioinformatics (Oxford, England) 26:139-40.

Starokozhko V, Abza GB, Maessen HC, Merema MT, Kuper F, Groothuis GMM. 2015. Viability, function and morphological integrity of precision-cut liver slices during prolonged incubation: Effects of culture medium. Toxicology in Vitro 30:288-299. DOI: 10.1016/j.tiv.2015.10.008.

Stoll LL, Spector AA. 1984. Changes in serum influence the fatty acid composition of established cell lines. In Vitro 20.

Su AI, Guidotti LG, Pezacki JP, Chisari F V, Schultz PG. 2002. Gene expression during the priming phase of liver regeneration after partial hepatectomy in mice. Proceedings of the National Academy of Sciences of the United States of America 99:11181-6. DOI: 10.1073/pnas.122359899.

Szalowska E, van der Burg B, Man H-Y, Hendriksen PJM, Peijnenburg AACM. 2014. Model Steatogenic Compounds (Amiodarone, Valproic Acid, and Tetracycline) Alter Lipid Metabolism by Different Mechanisms in Mouse Liver Slices. PLoS ONE 9:e86795. DOI: 10.1371/journal.pone.0086795.

Tocher DR. 2003. Metabolism and Functions of Lipids and Fatty Acids in Teleost Fish. Reviews in Fisheries Science 11:107-184. DOI: 10.1080/713610925.

Tocher DR, Bell JG, MacGlaughlin P, McGhee F, Dick JR. 2001. Hepatocyte fatty acid desaturation and polyunsaturated fatty acid composition of liver in salmonids: effects of dietary vegetable oil. Comparative Biochemistry and Physiology Part B: Biochemistry and Molecular Biology 130:257270. DOI: 10.1016/S1096-4959(01)00429-8.

Tu WC, Cook-Johnson RJ, James MJ, Mühlhäusler BS, Gibson RA. 2010. Omega-3 long chain fatty acid synthesis is regulated more by substrate levels than gene expression. Prostaglandins, Leukotrienes and Essential Fatty Acids 83:61-68. DOI: 10.1016/J.PLEFA.2010.04.001. 
582 Vancha AR, Govindaraju S, Parsa KV, Jasti M, González-García M, Ballestero RP. 2004. Use of 583 polyethyleneimine polymer in cell culture as attachment factor and lipofection enhancer. $B M C$ 584 Biotechnology 4:23. DOI: 10.1186/1472-6750-4-23.

585 Voss A, Reinhart M, Sankarappa S, Sprecher H. 1991. The metabolism of 7,10,13,16,19-

586 docosapentaenoic acid to $4,7,10,13,16,19$-docosahexaenoic acid in rat liver is independent of a 4587 desaturase. Journal of Biological Chemistry 266:19995-20000.

588 Wake K, Sato T. 2015. "The Sinusoid" in the Liver: Lessons Learned from the Original Definition by 589 Charles Sedgwick Minot (1900). The Anatomical Record 298:2071-2080. DOI: 10.1002/ar.23263.

590 Warburg O. 1923. Versuche an überlebendem Karzinomgewebe. Bioche- mische Zeitschrift 142:317-333.

591 Wu X, Roberto JB, Knupp A, Kenerson HL, Truong CD, Yuen SY, Brempelis KJ, Tuefferd M, Chen A, 592 Horton H, Yeung RS, Crispe IN. 2018. Precision-cut human liver slice cultures as an immunological 593 platform. Journal of Immunological Methods 455:71-79. DOI: 10.1016/J.JIM.2018.01.012. 


\section{Figure 1}

Viability and morphology of liver slices.

(A) Confocal scanning laser microscope (CLSM) image of a liver slice. Cells are stained with Hoechst (blue) and dead cells with propidium iodide (red). (B) Cell viability when incubated in media (none) and media supplemented with alpha-linolenic acid (ALA), insulin (INS), empty methyl- $\beta$-cyclodextrin (BCD), or a combination as measured by CLSM live/dead counts. Points represent viability measurements from a single liver slice. (C) Cross sections of paraffin embedded liver slice sampled at day zero (immediately after slicing), day three, and day five. The sections were stained with hematoxylin and eosin and photographed using light microscopy at 20x magnification. Scale bars are $100 \mu \mathrm{M}$ in length. 


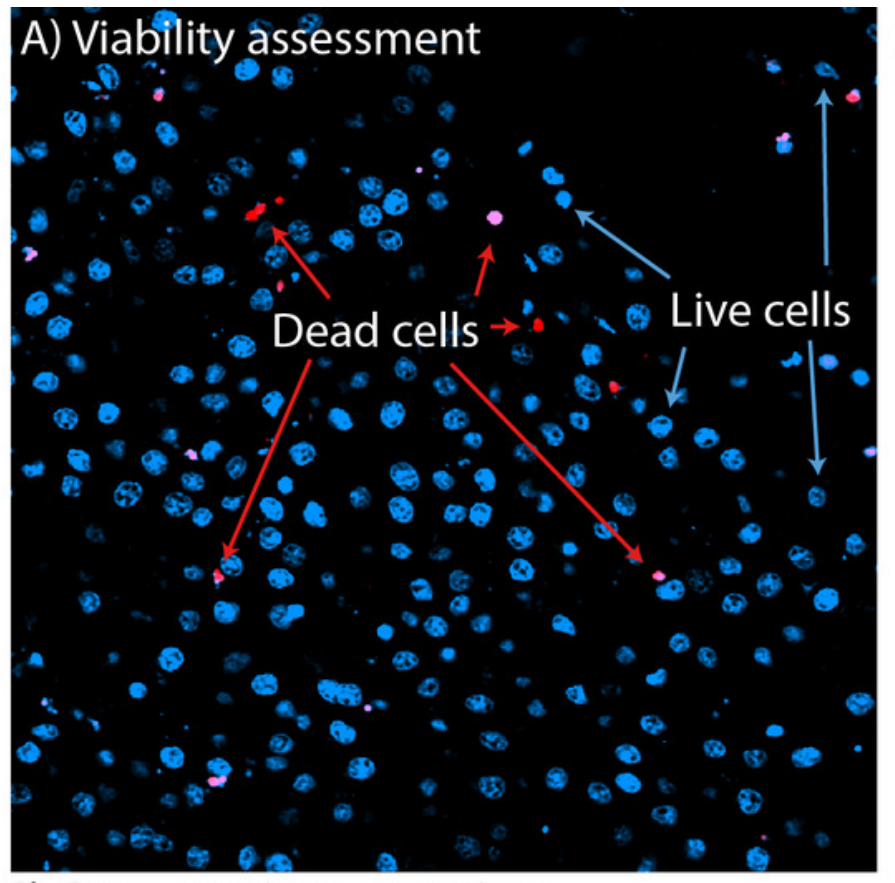

C) Cross-sections over time Day 0

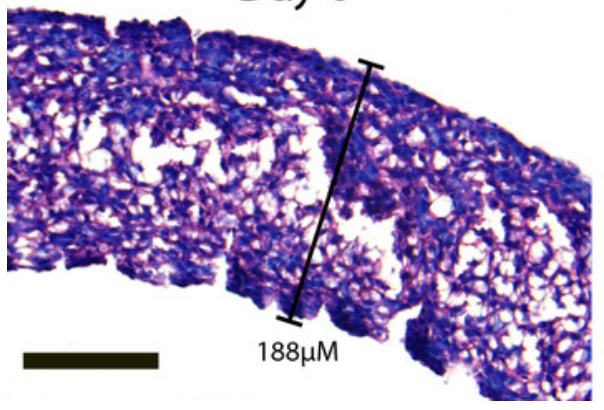

B) Viability under different conditions

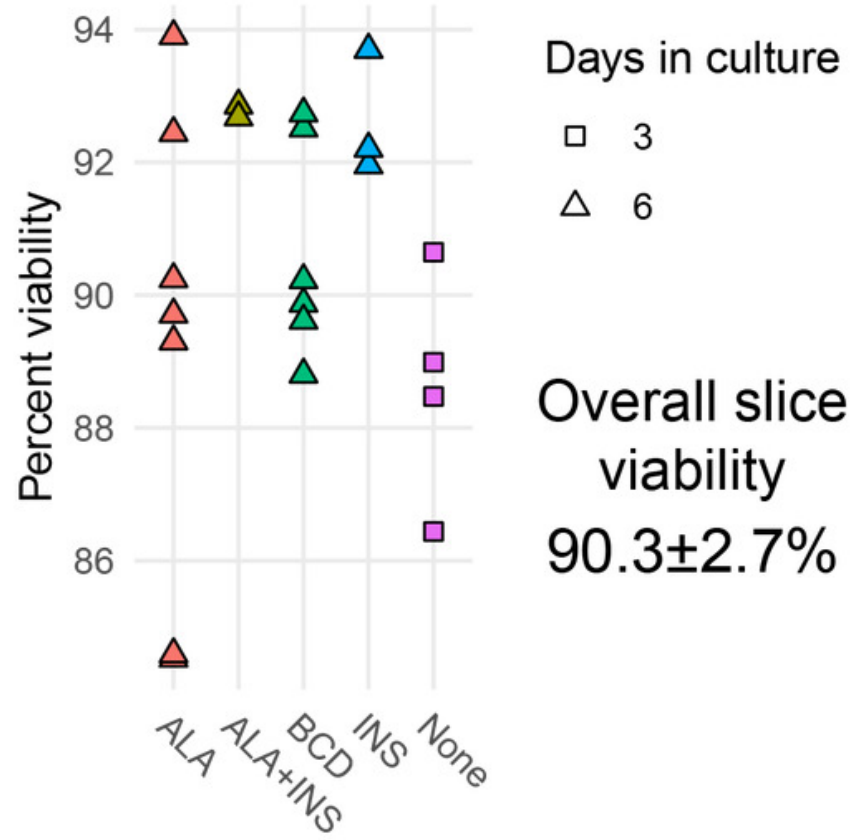

Day 3

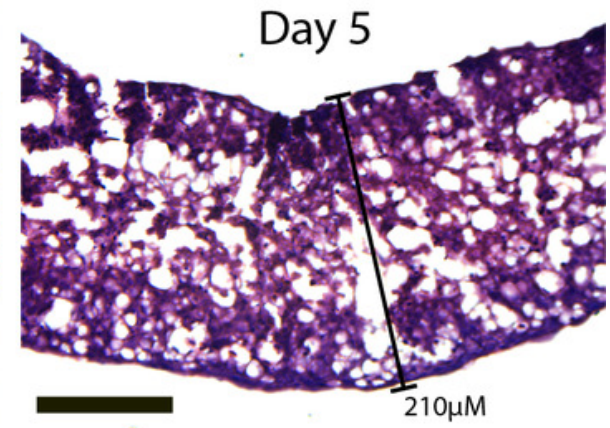




\section{Figure 2}

Global gene expression patterns over time.

A) Heatmap showing changes in the liver slice transcriptome over time. Heatmap includes 16,267 genes significantly differentially expressed (FDR $<0.01, \log 2 F C>1$ ) over the course of 9 days. Each time point was measured in triplicate. Transcript abundance is expressed in counts per million and were individually scaled across days to highlight changes in gene expression. B) Genes behaving similarly over time were clustered using Ward's method and broken into eight groups. Trend lines are based on mean scaled values in each cluster. C) KEGG pathway enrichment analysis was run on each cluster to determine how the liver slices are changing over time. Each point represents a significantly enriched pathway $(p<0.001)$. 


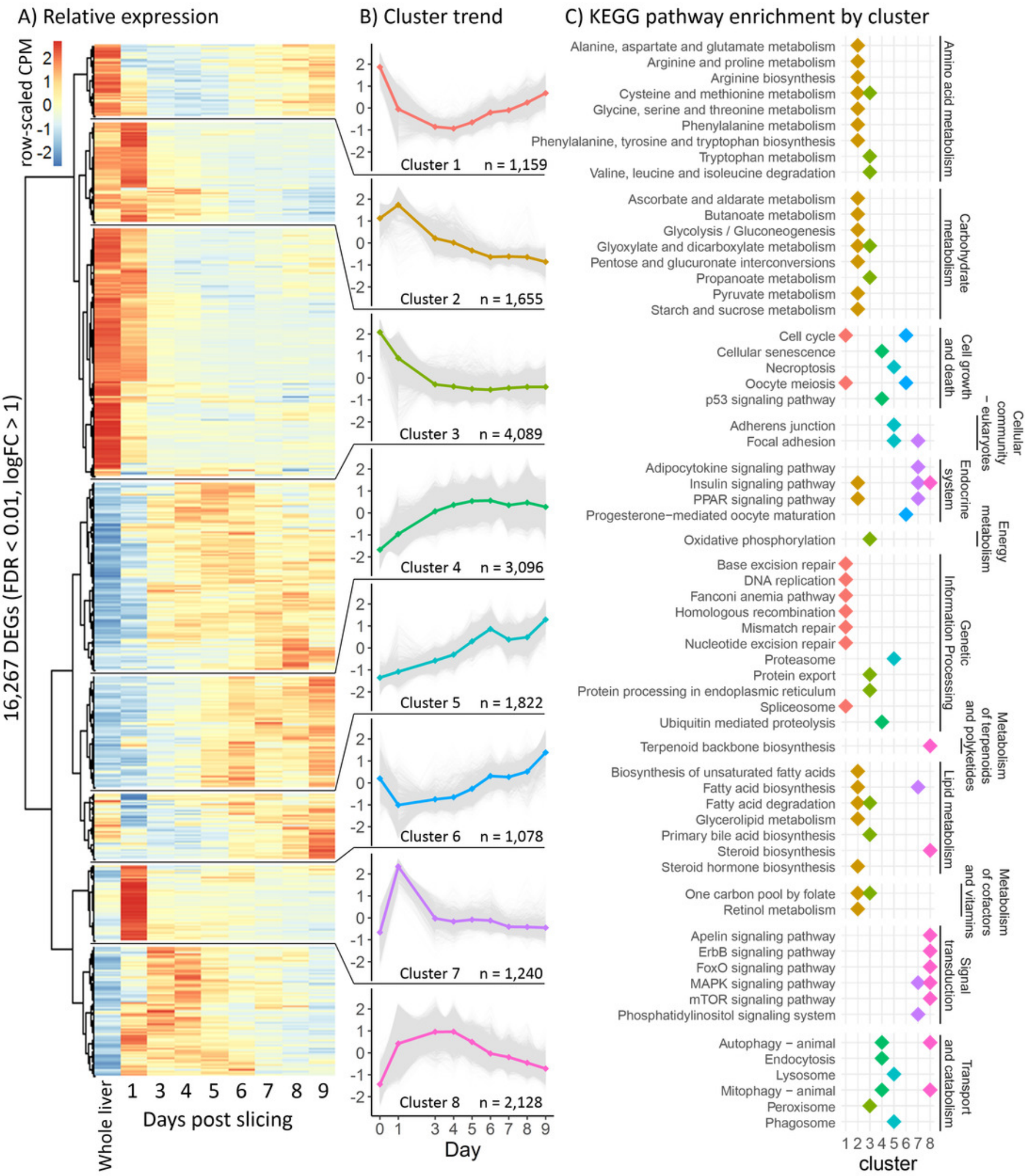




\section{Figure 3}

Co-expression correlation of liver slices over time for select lipid metabolism pathways.

Co-expression correlations (Spearman) between mean whole liver expression and gene expression from different days in time course one (red), time course two (green), and time course three (blue). Experiments use data from liver slices in triplicate each day.

Experiments were divided by insulin and media change regime. Time course two and three contained insulin, time course one and three had media changed every three days, and time course two had media changed daily. Correlations were calculated for all expressed genes (top left) and genes from seven lipid metabolism pathways.

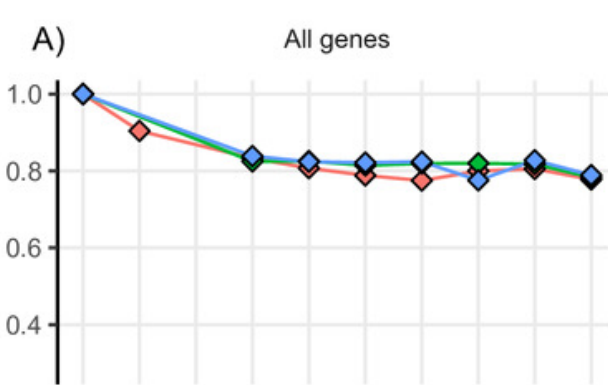

C)

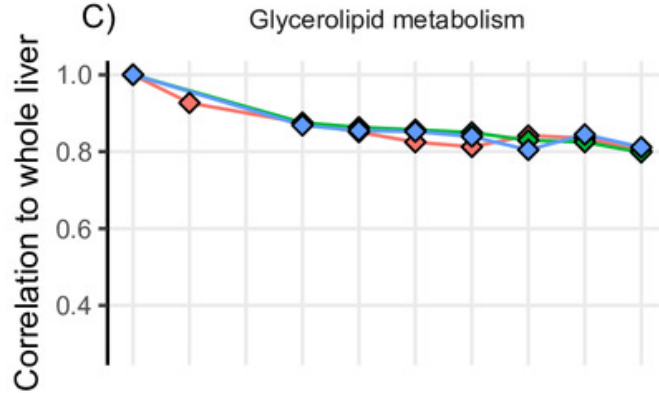

F) PPAR signaling

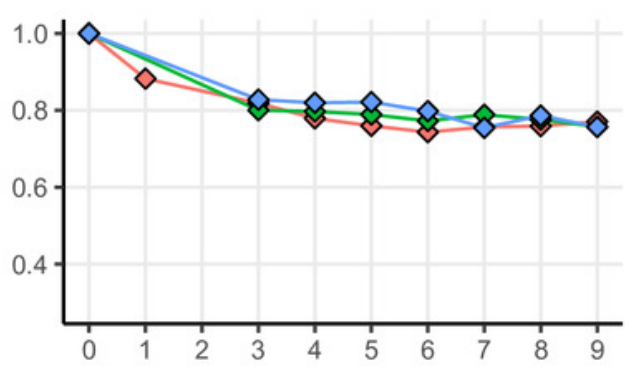

B)

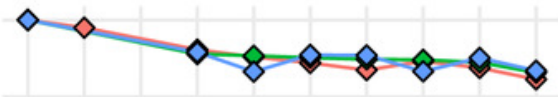

G) Glycerophospholipid metabolism

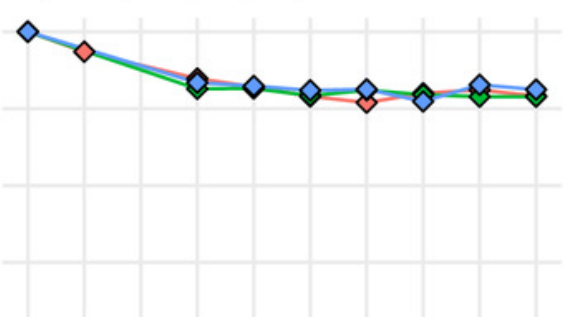

G)

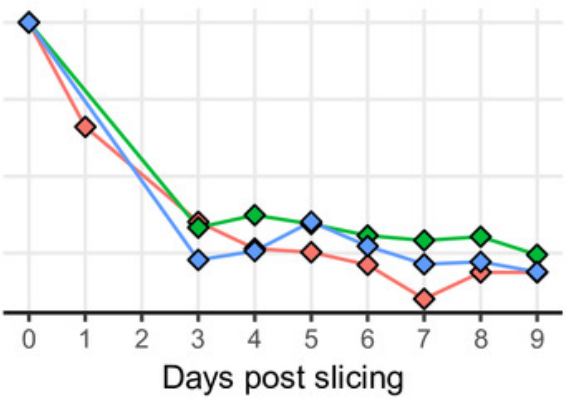

Experiment

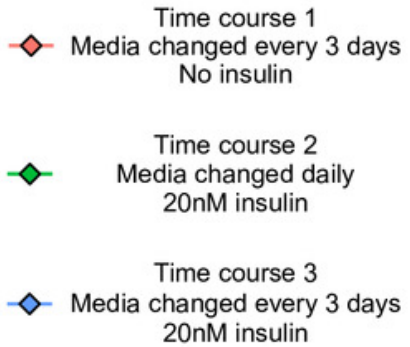

E)

Fatty acid biosynthesis

$\diamond$

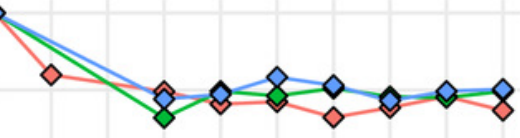

H) Insulin signaling

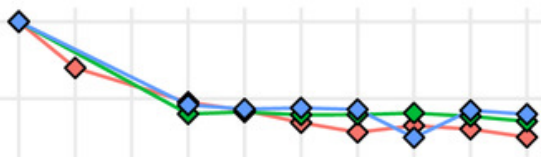




\section{Figure 4}

Effect of ALA and insulin on PUFA biosynthesis in liver slices.

A) Schematic diagram of the polyunsaturated fatty acid biosynthesis (PUFA) biosynthesis pathway. B) Gene-scaled log counts per million (CPM) of PUFA biosynthesis genes with increasing alpha-linolenic acid (ALA) concentration. C) Gene-scaled logCPM of PUFA biosynthesis genes with increasing insulin concentration with and without ALA supplementation. D) Relative abundance of ALA, 20:3n3, eicosapentaenoic acid, and docosahexaenoic acid with increasing ALA concentration. For all plots, large square, diamond, or circle points show mean scaled values of triplicate slices (logCPM or percent fatty acid) while small points show scaled values of individual replicates. Point size corresponds to unscaled values (logCPM or percent fatty acid) of the mean. Letters indicate significant $(q<0.05)$ differences between groups (ALA or insulin concentration) for corresponding genes or fatty acids. 
A) PUFA biosynthesis pathway

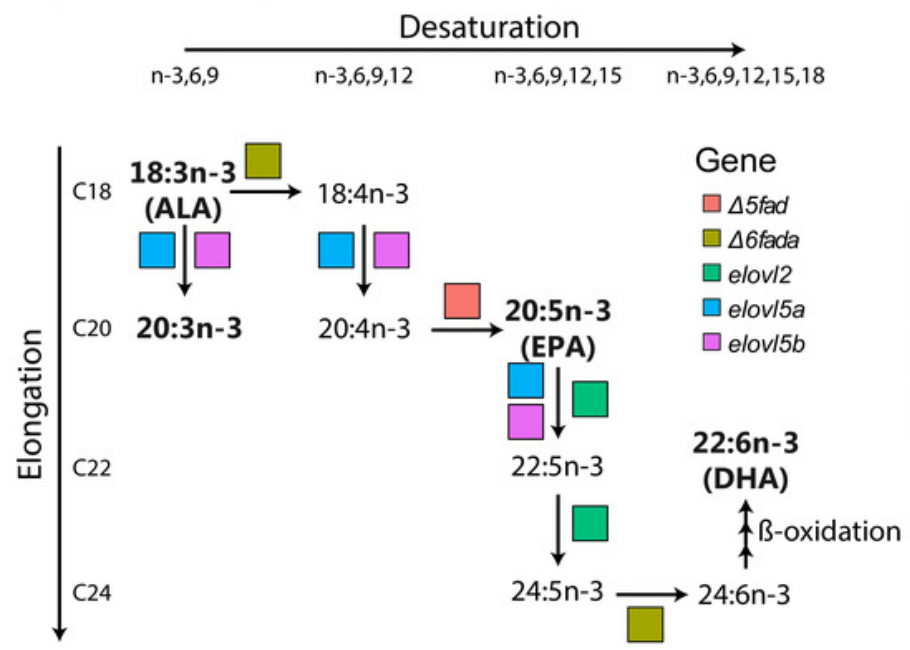

C) Fatty acid shifts from ALA supplementation

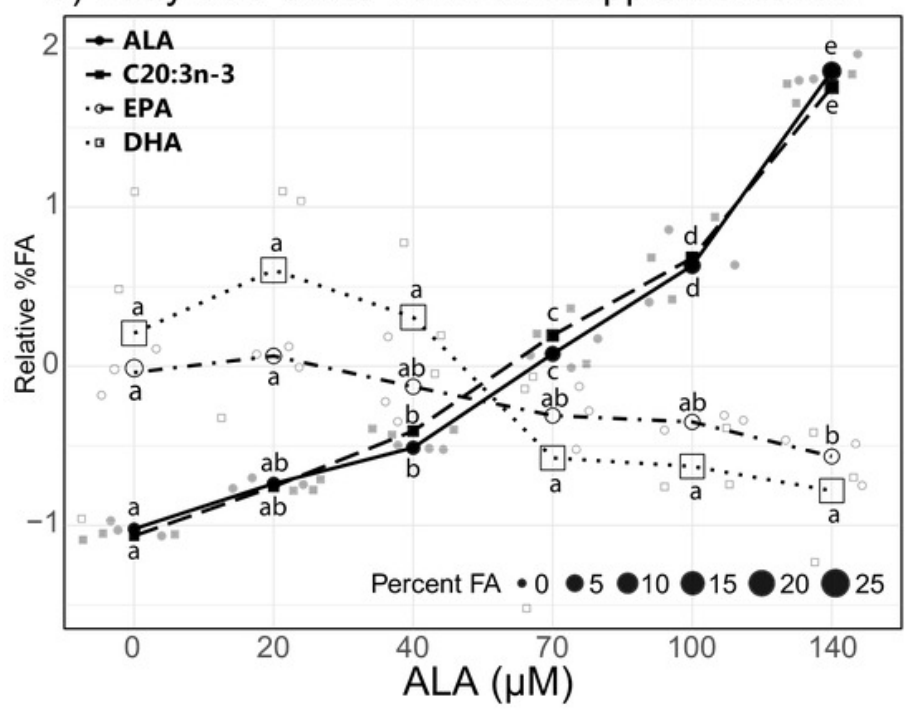

B) Expression response to ALA supplementation

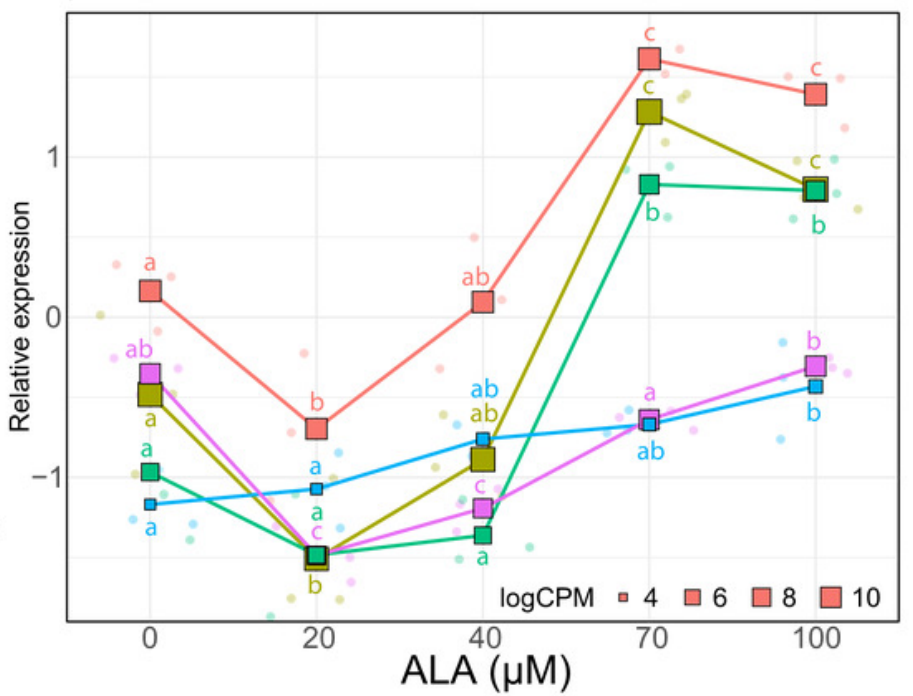

D) Expression interaction between ALA and insulin

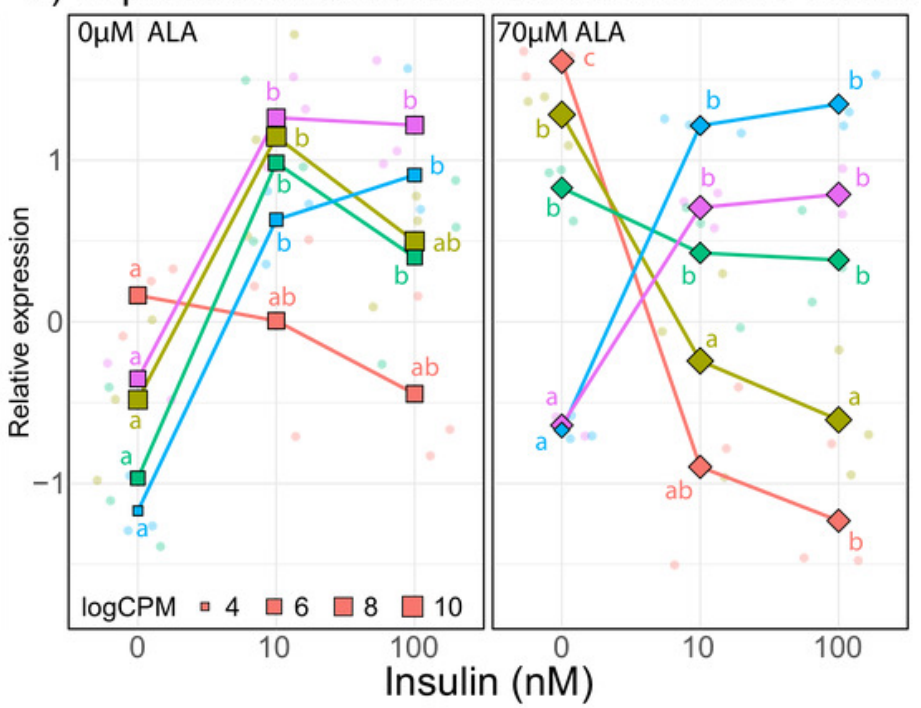




\section{Figure 5}

Expression of select pathways and genes in hepatocyte culture, liver slice culture, and whole liver.

Transcriptomic data from each source was pooled to give a range of possible gene expression levels (hepatocyte culture $n=16$, liver slice culture $n=89$, whole liver $n=210$ ) A) Relative expression profiles for five selected lipid metabolism pathways. Values are expressed mean scaled log2 CPM of all genes within a pathway for each sample with a minimum CPM of 1. B) Relative expression of key genes in the PUFA biosynthesis pathway with a minimum CPM of 10. Abbreviations: $\Delta 6 \mathrm{fad}=$ delta -6 fatty acid desaturase,elovl$/ 5=$ fatty acid elongase 5, $45 \mathrm{fad}=$ delta-5 fatty acid desaturase,elov/2 = fatty acid elongase 2. C) Relative expression of select liver marker genes with a minimum CPM of 10. Abbreviations:alb= albumin, $g 6 \mathrm{pcl}=$ glucose- 6 -phosphatase-like, tat $=$ tyrosine aminotransferase-like,cyp3al= cytochrome P450 3A27-like,pck1 = phosphoenolpyruvate carboxykinase 1,tdo2= tryptophan 2,3-dioxygenase,tod2l= tryptophan 2,3-dioxygenase-like. 


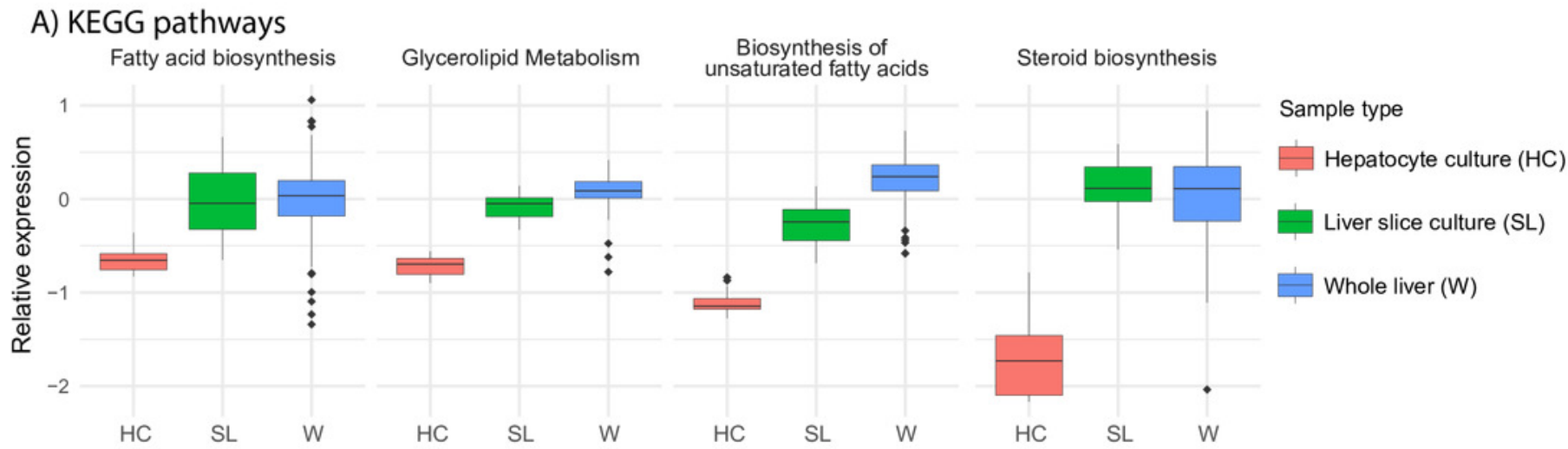

B) PUFA biosynthesis genes

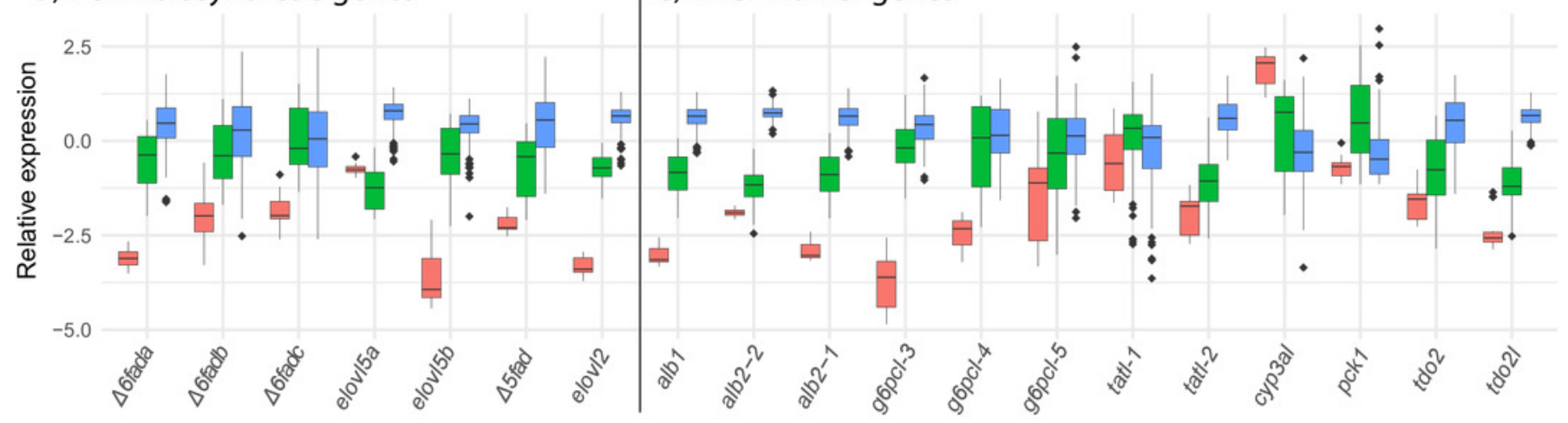

